\title{
The Antimicrobial Peptides: Ready for Clinical Trials?
}

\author{
Michael AB Naafs* \\ Naafs International Health Consultancy, Europe
}

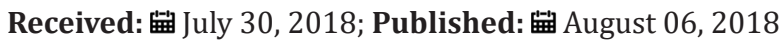

*Corresponding author: Michael AB Naafs, Internist-endocrinologist with a long clinical career in internal medicine and endocrinology, Naafs International Health Consultancy, Europe

\begin{abstract}
Bringing the antimicrobial peptides, AMPs, in pharmaceutical business was a long process with many technical hurdles after their discovery more than 30 years ago. Structure, classification and mode of action of the AMPs as well as the selection of AMPs for clinical use are discussed. The preclinical and clinical trial results in phase 1 and phase 2 studies are discussed for 9 AMPS. These results are encouraging for the future of the AMPs
\end{abstract} as alternative antibiotics.

Abbreviations: AMPs: Antimicrobial Peptides; LPS: Lipopolysaccharides; MIC: Minimum Inhibitory Concentration; MDR: Multidrug Resistance; CFDA: China Food and Drug Administration; IDRs: Innate Defense Regulators

\section{Introduction}

Antimicrobial peptides (AMPs) are short proteins with antimicrobial activity. A large portion of known AMPs originate from insects. AMPs from insects represent a potential source of alternative antibiotics in the face of rising antibiotic resistance. AMPs can act against bacteria, viruses, fungi or parasites. In insects they contribute to host innate immunity [1-4]. The first AMP was extracted in 1980 from the pupae of the giant silk moths and its bactericidal properties were demonstrated for the first time [5]. Now more than 1500 AMPs have been identified in different organisms including plants, fungi, bacteria and animals. Insects are the primary source of AMPs. In traditional Chinese medicine over 1700 medicines have been produced from circa 300 insect species [6]. Difficulties in species identification, drug toxicity, development costs and large scale production slow the development of insect products into potential modern medicines [7].

\section{Structure and Classification}

The increasing number of published insect genomes and transcription datasets combined with the ability to probe hemolymph samples directly using proteomics techniques has resulted in the discovery of many new AMPs in the past few years $[8,9]$. Novel AMPs can be identified by homology to known peptides, but also by other features such as the presence of protease cleavage sites and expression profiles that focus on immunocompetent cells and tissues $[10,11]$.

Insect AMPs can be classified according to their structure or function. The three major structural classes are linear alpha- helical peptides without cysteine residues, peptides with a beta-sheet globular structure stabilized by intra-molecular disulfide bridges and peptides that contain unusually high numbers of specific amino acid residues such as proline or glycine $[1,12,13]$.

Cecropin is the prototype of alpha-helical linear AMP [5]. It is active against gram-negative bacteria as Escherichia coli. Other cecropins have been identified recently as well as cecropin-like peptides as sarcotoxins, hyaphyncin and enbocin, which can act synergistically against both gram-negative and gram-positive bacteria [14]. Defensins are the prototype of the second major structural class of insect AMPs. They have the predominantly betasheet globular structure. Most insect defensins act against grampositive bacteria. Some inhibit gram-negative bacteria [15].

The third structural group are the proline-rich AMPs. Examples of these are the drosocins, lebocins, metchnikowins and apidaecins. They are divided in short-chain (fewer than 20 proline residues) or long-chain (more than 20 proline residues) AMPs. The former are more potent against gram-negative bacteria whereas the latter are more active against gram-positive bacteria and fungi $[16,17]$. Such differences in their specificity may be mediated by their distinct lipopolysaccharide binding activity or their ability to penetrate bacterial membranes $[18,19]$. The attacins and gloverins belong also to the third structural group and form the glycine-rich AMPs. Attacin from Hylophora cecropia inhibits outer membrane synthesis in E. coli whereas from Manida sexta binds to Grampositive membrane liposaccharides [20-22].

\section{Mode of Action}

Most AMPs are cationic molecules which perturb the target cells through the formation of ion channels or transmembrane pores and in this way destroy the bacterial cell $[23,24]$. The main targets 
of AMPs are lipids in cell membranes. The AMPs bind to anionic phospholipids and phosphate groups of lipopolysaccharides (LPS) of gram-negative bacteria as well to teichoic acid and lipoteichoic acids composing the peptoglycidan layer of gram-positive bacteria. The peptide anchors in the cytoplasmic membrane of the microorganism and changes the membrane structure which facilitates the incorporation into the phospholipid dual layer of the cytoplasmic membrane [25]. After adsorption into the membrane surface AMP scan induce a variety of membrane perturbations within tens of nanoseconds [26].

For most AMPs an early event is the formation of hydrogen bounds between the basis residues (eg. arginine and lysine) and the phosphate groups of the lipids. Both arginine and lysine are hydrogen bond donors. However, arginine can form more stable bidentate hydrogen bounds with phosphate groups. Hydrophobic residues can further penetrate and disorganize the lipid tail region of the membrane. As more AMPs accumulate at the membrane-water interface the membrane becomes thin [27]. Membrane thinning results in lateral expansion affecting the mechanical properties of the membrane and the electrostatic properties ultimately leading to cell death. This is the pore or toroidal model Currently 4 different models of possible actions of AMPs on bacterial cell membranes are described. Beside the toroidal model the second model is called the carpet model. In this model the AMPs cover the cell membrane in a carpet-like manner. This action requires high AMPs concentrations and causes cell membrane dissolvance, similar to the action of a detergent. An example of AMPs acting in this way are the cecropins.

The third model for AMPs action is the "barrel-stave" model, in which peptides bind to the cell membrane and insert themselves into the hydrophobic core of the membrane, forming a pore and causing a leakage of cytoplasmic material and a decrease in membrane potential. In this way membrane damaging peptides such as gramicidin, finally kill the cell. The fourth and the last strategy is the destruction of cell membranes by creating "ünstructured ring pores" ie. aggregate channels $[23,25]$. Apart from the membrane destruction some AMPs like pyrrhocoricin, drosocin and apidaecin may exert antibacterial activity by interactions with intracellular targets thus disrupting intracellular processes. These belong to the short proline-rich AMPs.

At first the peptides permeate and traverse the outer membrane and enter the periplasmic space. Then the process looks for the stereospecific and irreversible translocation into the cytoplasma of the bacterial cell. Inside the cell they interact with the target which is mainly the $70 \mathrm{kDa}$ heat shock protein DnaK. They can also interfere with DNA and RNA synthesis and have been shown to target E. coli, Kliebsiella pneumoniae, Pseudomonas aeruginosa and Acinetobacter baumannii [28]. Because of the contribution of electrostatic interactions binding of AMPs to microbial membranes is significant while binding of AMPs to the neutral phosphatidylcholine/cholesterol sfingomyeline-rich surfaces of animal plasma membranes is weaker. AMPs can dissipate the electrochemical gradient across microbial plasma membranes within a few seconds of addition [29,30].
AMPs must be able to rapidly pass through the thick protoglycean layer of Gram-positive bacteria and the outer lipopolysaccharide (LPS) layer of gram-negative bacteria to reach the cytosolic inner membrane. Permeation of larger markers including dye markers, metabolites and cytosolic proteins through the cytoplasmic membrane occurs on the time scale of minutes to ten minutes [29]. After an hour or more in contact with AMs gross disruption of microbial membrane structure and morphology is often noted including blebbing, vesiculation, fragmentation, release of DNA, cell aggregation and cell death. This is in sharp contrast to conventional antibiotics which are largely bacteriostatic and need hours to days to exert their effects.

\section{Therapeutic Potentials of AMPs}

Due to complex and multitarget mechanisms of action AMPs are distinguishable from conventional antibiotics and make them an ideal candidate to generate new antibiotics [31]. They have synergistic effects with conventional antibiotics, relatively small size, neutralizing endotoxin ability, considerable low minimum inhibitory concentration (MIC) and show that they are highly prone to kill bacteria. They have potent activity against biofilms too which also showed resistance to traditional antibiotics. In addition, AMPs have shown a wide range of antiviral properties in vitro [32].

In malignant cancer cells multidrug resistance (MDR) is a major mechanism of drug resistance. Few AMPs displayed cytotoxicity against MDR cancer cells. They were able to kill cancer cells rapidly, had lower side effects with easier absorption [33]. For their therapeutic use in cancer they are mandatory to perform specific anticancer activity alongside stability in serum [34,35]. A better understanding in this area would definitely contribute to cancer research.

\section{Selecting AMPs for Clinical Use}

Bringing emerging peptides in the pharmaceutical business is a long way with many hurdles [36]. It is not surprising that the AMPs are no exception in this way. Despite the advantages of AMPs progress to date in developing them for clinical use has been disappointing. The main advance has been with vertebrate AMPs for use in topical applications and a few AMPs have entered clinical trials $[7,13,37,38]$. These AMPs were designed for a number of external uses as skin care, acne, eye infections and catheterrelated pathogens. There have been a number of reasons for the slow progress in AMPs becoming available for clinical use. Lack of interest by large pharmaceutical companies in antibiotics for more than 30 years. Small niche biopharma companies are taking the lead fortunately.

High production costs have always been a major hurdle to the development of the AMPs since they only occur at low concentrations and the cost of solid phase synthesis is very high. Truncated synthetic AMP analogues can be produced at cheaper costs [36]. Recombinant technology had to be modified because of the antibacterial activity of the AMPs and their proteolytic degradation during production. Recent studies however have used cost effective modified recombinant techniques with $E$. coli or with the methylotrophic yeast Pichia pistores as vectors to produce fully 
functional insect cecropins capable of killing a range of bacteria, including MRSA [39-41].

There are also concerns about the stability and toxicity towards mammalian cells $[42,43]$. With amino acid substitutions, sequence splicing and changes in ratios of hydrophobic amino acids now truncated designer compounds can be produced that are effective at very low MICs [44]. Regarding development of resistance of bacteria to AMPs this was thought to be less likely to occur than with conventional antibiotics as AMPs have multiple sites of action within the bacterial cell and involve fundamental changes in the membranes. However, resistance has been described, including resistance to insect melittin and cecropin, probably due to continual selection in the laboratory. Conditions in nature are very different [45-49].

\section{Amps in Preclinical and Clinical Trials}

In the last 30 years continuous efforts have been made to develop AMPs as clinically useful antimicrobials. To date no designed AMP antibiotics have yet reached the clinic. A number of AMPs and AMP derivates are already at the preclinical stage and in clinical trials.

PL-5 is an alpha helical AMP and is developed by Prote Light Pharmaceuticals and has recently obtained approval from the China Food and Drug Administration (CFDA) to enter clinical trials for skin infections. Pl-5 is the first AMP to enter the clinical stage in China. PL-5 is a low toxicity and highly potent AMP against a broad spectrum of drug-resistant bacteria. In addition PL-5 is able to synergize with conventional antibiotics to improve antibacterial activity in vitro and in vivo against both Gram-positive and Gram-negative bacteria. This may help or delay the emergence of antibiotic resistance [50].

POL 7080 is a synthetic cyclic peptide derived from protegin 1. POL 7080 is active against Gram-negative and Gram-positive bacteria and works by inhibiting a homolog of the beta-barrelprotein LptD. LptD is an outer membrane protein widely distributed in Gram-negative bacteria that functions the assembly of LPS in the outer membrane. POL 7080 is highly active on a broad panel of clinical isolates including multi-drug resistant Pseudomonas with outstanding in vivo efficacy in septicemia, lung and thigh models. (Polyphar) POL 7080 is developed by Polyphar Ltd. and has completed a phase 1 trial with its partner Roche. POL 7080 has also completed a phase 2 trial in 20 patients with exacerbations of noncystic fibrosis bronchiectasis in 2015 [51]. To date the structure of POL 7080 has not been revealed.

DPK 060 is a cationic peptide that has recently completed a phase 2 study of topical application for atopic dermatitis. DPK 060 is a broad spectrum cationic peptide active against both Grampositive and Gram-negative bacteria. Similar to other AMPs DPK 060 is also membrane targeting [52]. DPK 060 is developed by Pergamum AB. The results of a phase 2 clinical trial of DPK 060 in outer ear infections showed a statistically significant improvement in a 10 day cure rate compared to placebo and that DKP 060 is safe and tolerable [53]. Pergamum AB has also developed LL-37, a human cathelicidin subunit. LL-37 is active against both Gram- negative and Gram-positive bacteria. LL-37 is developed for the treatment of chronic leg ulcers. The clinical phase results show that LL-37 has a significant improved healing rate compared to placebo [53].

\section{Innate Defense Regulators (IDRs)}

IDRs are a novel class of synthetic peptides that enhance the control of microbial infections. IDRs do not impact the adaptive immune system and do not interfere with chemotherapy, radiation or antibiotic treatments. Disquietude is a fully synthetic 5-amino acid peptide derived from indolizidine with high aqueous solubility and stability. SGX 942 (Soligenix) has broad spectrum activity against Gram-negative and Gram-positive bacteria and complements the actions of standard care antibiotics [54]. SGX 942 was first developed by Inimex and is currently pursued in a phase 2 trial by Soligenix as a treatment for oral mucositis [51]. SGX 942 has been awarded Fast Track designation from the FDA for the treatment of oral mucositis as a result of radiation and/or chemotherapy in head and neck cancer patients.

Brilacidin is a small molecule arylamide- mimic of AMPs that shows potent antimicrobial activity against a wide range of multidrug-resistant Gram-negative and Gram-positive bacteria. Brilacidin was first developed by Polymedix. Inc. and is now purchased by Cellceutix Corp. Brilacidin has completed phase $2 \mathrm{a}$ and phase $2 \mathrm{~b}$ trials for the treatment of acute $S$. aureus skin infections. Similar to other AMPs brilacidin is a membrane targeting antimicrobial. It causes membrane disruption and shows efficacy in a MRSA keratitis model when applied topically; Brilacidin is equally effective as daptomycin and vancomycin [55].

Cellceutix is also developing CTIX 1278 (structure not revealed) a defensin- mimetic compound against the drug resistant superbug Klebsiella pneumoniae. CTIX 1278 is efficacious in a thigh burden study in a mouse model. The results are encouraging as CTIX 1278 shows similar efficacy compared to carbapenem. 6 LTX 109 is developed by Lytix Biopharma which focus on topical treatment of skin infections and nasal eradication of S. aureus. LTX 109 is a synthetic antimicrobial peptide mimetic which has completed phase 2 trials for the treatment of impetigo. LTX 109 is active against a broad range of bacteria including E. coli and S. aureus. LTX 109 is also active against a panel of drug resistant Gram-positive bacteria such as MRSA, vancomycin-intermediate resistant, daptomycin resistant and linezolid resistant strains [56].

Exeporfinium chloride (XF 73) is a synthetic diatonic porphyrin derivative being developed by Destiny Pharma, Brighton, UK. It has been evaluated in phase 1-2 trials for the prevention of post-surgical nasal staphylococcal infections. XF 73 is a photosensitizer that has broad spectrum activity against Gram-positive and Gram-negative bacteria and Candida albicans [57]. Similar to AMPs interaction of XF 73 with the cytoplasmic membrane of S. aureus is lethal. XF 73 showed no drug resistance for 4 common MRSA strains.

\section{Conclusion}

The research of the antimicrobial peptides, AMPs, has been very slow in the more than 30 years since their discovery. In the face of multidrug resistance to conventional antibiotics they have 
the potential to be an outstanding alternative. Lack of interest and responsibility of the large pharmaceutical companies, the high production costs and concerns about unknown safety and toxicity of oral administration, delay the introduction in clinical use. Small niche biopharma companies fortunately take the lead. At least the AMPs are now in the stage of phase 1 and phase 2 trials for topical applications as skin infections, chronic leg ulcers, wound infections and ear and eye infections. These results are discussed. and they show the future of the AMPs is still bright.

\section{References}

1. Bulet P, Stocklin R, Menin l (2004) Antimicrobial peptides: From invertebrates to vertebrates. Immunol Rev 198: 169-184.

2. Vale N, Aguiar L, Gomes P (2014) Antimicrobial peptides: A new class of antimalarial drugs? Front Pharmacol 5: 275

3. Hancock RE, Sahl HG (2006) Antimicrobial and host-defense peptides as new anti-infective therapeutic strategies. Nat Biotechnol 24(12): 15511557.

4. Brogden KA (2005) Pore formers or metabolic inhibitors in bacteria? Nat Rev Microbiol 3(3): 238-250.

5. Hultmark D, Steiner H, Rasmuson T (1980) Insect immunity: Purification and properties of three inducible bactericidal proteins from hemolymf of immunized pupae of Hydrophera cecropia. Eur J Biochem 106(1): 7-16.

6. Srivastava SK, Babau N, Pandey KC (2009) Traditional insect bioprospecting as human food and medicine. Indian J Traditional Knowledge 8(4): 485-494.

7. Ratcliffe NA, Mello CB, Garcia ES (2011) Insect natural products and processes: New treatments for human disease? Insect Biochem Mol Biol 41(10): 747-769.

8. Altincicek B, Vilcinskas A (2007) Analysis of the immune-related transcriptome from microbial stress resistant rat-tailed maggots of the drone fly Enstalis tenax. BMC Genomics 8: 326.

9. Vilcinskas A (2013) Evolutionary plasticity of insect immunity. J Insect Physiol 59(2): 123-129.

10. Vogel H, Altincicek B, Glóckner G (2011) A comprehensive transcriptome and immune-gene repertoire of the Lepidopteran model mini-host Galleria mellonella. BMC Genomics 12: 308.

11. Vicinskas A (2011) Anti- infective therapeutics from the Lepidopteran model host Galleria mellonella. Curr Pharm Design 17(13): 1240-1245.

12. Bulet P, Stocklin R (2005) Insect antimicrobial peptides: Structures, properties and gene regulation. Protein Pept Lett 12(1): 3-11.

13. Wiesner J, Vilcinskas A (2010) Antimicrobial peptides: The ancient arm of the human immune system. Virulence 1(5): 440-464.

14. Poppel AK, Vogel H, Wiesner J (2015) Antimicrobial peptides expressed in medicinal maggots of the blow fly Lucilia sericeta shows combinatorial activity against bacteria. Antimicrob Agents Chemother 59(5): 25082514

15. Langen G, Imani J, Altincicek B (2006) Transgenic expression of gallerimycin, a novel antifungal insect defensin from the greater wax moth Galleria Mellonella, confers resistance to pathogenic fungi in tobacco. Biol Chem 387(5): 549-557.

16. Rahnamaeian M, Langen G, Imani J (2009) Insect peptide metchnikowins confers on barley a selective capacity for resistance to fungal ascomycetes pathogens. J Exp Biol 60(14): 4105-4114.

17. Rahnamaeian M, Vilcinskas A (2012) Defense gene expression is potentiated in transgenic barley expressing antifungal peptide Metchnikowin throughout powdery mildew challenge. J Plant Res 125(1): 115-124.
18. Taniguchi M, Ochai A, Kondo H (2015) Pyrrhocorricin, a proline-rich antimicrobial peptide derived from insect, inhibits the translation process in the cell-free Escherichia coli protein synthesis system. J Biosc Bioeng 121(5): 591-598.

19. Rao XJ, Xu XX, Yu XG (2012) Functional analysis of two lebocin-related proteins from Manduca sexta. Insect Biochem Mol Biol 42(4): 231-239.

20. Carlsson A, Engstrom P, Palva ET (1991) Attacin, an antibacterial protein from Hyadophora cecropia inhibits synthesis of outer membrane proteins in Escherichia coli by interfering with omp gene transcription. Infect Immun 59(9): 3040-3045.

21. Carlsson A, Nystrom T, De Cock H (1998) Attacin: An insect immune protein binds LPS and triggers the specific inhibition of bacterial outermembrane protein synthesis. Microbiology 144(8): 2179-2188.

22.Xu XX, Zhong X, Yi HY (2012) Manduca gloverin binds microbial components and is active against bacteria and fungi. Dev Comp Immunol 38(2): 255-284.

23. Duclohier H (2012) How do channel- and pore forming helical peptides interact with lipid membranes and how does this account for their antimicrobial activity? Mini-Rev Med Chem 2: 331-342.

24. Park Y, Hahm KS (2005) Antimicrobial peptides (AMPs): Peptide structure and mode of action. J Biochem Mol Biol 38(5): 507-516.

25. Zylawska M, Wyszynska A (2011) Antimicrobial peptides: Defensins. Post Mikrobiol 50: 223-234.

26. Li J, Liu S, Lakshminrayanan P (2013) Molecular simulations suggest how a branched antimicrobial peptide perturbs a bacterial membrane and enhances permeability. Biochim Biophys Acta Biomembranes 1828(3): 1112-1121.

27. Sato H, Feix JB (2006) Peptide-membrane interactions and mechanisms of membrane destruction by amphipathic alpha-helical antimicrobial peptides. Biochim Biophys Acta Biomembranes 1758(9): 1245-1256.

28. Hansen A, Scháfer I, Knappe D (2012) Intracellular toxicity of prolinerich antimicrobial peptides shuttled into mammalian cells by the cellpenetrating peptide-penetration. Antimicrob Agents Chemother 56(10): 5194-5201.

29. Rathinakumar R, Walkenhorst WF, Wimley WC (2009) Combinatorial Design and High-throughput Screening: The Importance of Interfacial Activity. J Am Chem Soc 131(22): 7609-7617.

30. Rathinakumar R, Wimley WC (2008) Biomolecular engineering by combinatorial design and high-throughput screening, small, soluble peptides that permeabilize membranes. J Am Chem Soc 130(30): 98499858.

31. Hancock RE, Nijnik A, Philpott DJ (2012) Modulating immunity as a therapy for bacterial infections. Nature Rev Microbiol 10(4): 243-254.

32. Steckbeck J, Deslouches B, Montelaro R (2013) Antimicrobial peptides: New drugs for bad bugs? Exp Opin Biol Ther 14(1):11-14.

33. Parachin NS, Franco OL (2014) New edges of antibiotic development: Antimicrobial peptides and corresponding resistance. Front Microbiol 5: 147.

34. Giuliani A, Pirri G, Nicoletto S (2007) Antimicrobial peptides: An overview of a promising class of therapeutics. Open Life Sciences 2(1): $1-33$.

35. Chen Y, Xu X, Hong S (2001) RGD-tachyplesin inhibits tumor growth. Cancer Res 61(6): 2334-2338.

36. Uhlig T, Kyprianou T, Martinelli FG (2014) The emergence of peptides in the pharmaceutical business: From exploration to exploitation. EuPA Open Proteomics 4: 58-69.

37. Peravali JB, Kotara SR, Sobha K (2013) Antimicrobial peptides: An effective alternative for antibiotic therapy. Mintage J Pharm Med Sci 2(2): 1-7. 
38. Zarou M (2007) Multifunctional antimicrobial peptides: Therapeutic targets in several human diseases. J Mol Med 85(4): 317-329.

39. Bommarius B, Jenssen H, Elliot M (2010) Cost-effective expression and purification of antimicrobial and host defense peptides in Escherichia coli. Peptides 31(11): 1957-1965.

40. Wang H, Meng XL, Xu JP (2012) Production, Purification and characterization of the cecropin from Plutella xylostella: pxCECA1 using an intein-induced self-cleavable system in Escherichia coli. Appl Microbiol Biotechnol 94(4): 1031-1039.

41. Wang X, Zhu M, Yang G (2011) Expression of cecropin B in Pichia pastoris and its bioactivity in vitro. Exp Ther Med 2(4): 655-660.

42. Kang SJ, Kim DH, Mishig-Ochir T (2012) Antimicrobial peptides: Their physicochemical properties and therapeutic applications. Arch Pharm Res 35(3): 409-413.

43. Ntwasa M, Goto A, Kureta S (2012) Coleopteran, antimicrobial peptides: Prospects for clinical applications. Int J Microbiol 101989: 1-8.

44. Huang JJ, Lu JC, Lu M (2012) The design and Construction of K11: A novel alpha-helical antimicrobial peptide. Int J Microbiol 764834: 1-6.

45. Fehri LF, Strand-Pugnet P, Gourgues G (2005) Resistance to antimicrobial peptides and stress response in Mycoplasma pulmonis. Antimicrob Agents Chemother 49(10): 4145-4165.

46. Giacometti A, Cirioni O, Barchiesi F (1999) In-vitro activity of cationic peptides alone or in combination with clinically used agents against Pseudomonas aeruginosa. J Antimicrob Chemother 44(5): 641-645.

47. Perron GG, Zasloff M, Bell G (2006) Experimental evolution of resistance to an antimicrobial peptide. Proc Royal Soc Biol Sci 273(1583): 251-256.

48. Chernysh SJ, Gordja NA (2011) The immune system of maggots of the bow fly (Calliphora vicina) as a source of medicinal drugs. J Evol Biochem Physiol 47(6): 524-531.
49. Park SE, Park Y, Hahm KS (2011) The role of antimicrobial peptides in preventing multidrug-resistant bacterial infections and biofilm formation. Int J Mol Sci 12(9): 5971-5992.

50. Feng Q, Huang Y, Chen M (2015) Functional synergy of alpha-helical antimicrobial peptides and traditional antibiotics against Gram-negative and Gram-positive bacteria in vitro and in vivo. Eur J Clin Microbiol Infect Dis 34(1): 197-204.

51. Butler MS, Blaskovich MA, Cooper MA (2017) Antibiotics in the clinical pipeline at the end of 2015. J Antibiot 70(1): 3-34.

52. Harvey AL, Edrada-Ebel P, Quinn R (2015) The re-emergence of natural products for drug discovery in the genomics era. Nat Rev Drug Discov 14(2): 111-129.

53. Lee DK, Bhunia A, Kotler SA (2015) Detergent-type membrane fragmentation by MSI-78, MSI-367, MSI-594 and MSI-843 antimicrobial peptides and inhibition by cholesterol: A solid state nuclear magnetic resonance study. Biochem 54(10): 1879-1907.

54. North JR, Takenaku S, Rozek A (2016) A novel approach for emerging and antibiotic resistant infections: Innate defense regulators as an agnostic therapy. J Biotechnol 226: 24-34.

55. Kowalski RP, Romanowski EG, Yates KA (2016) An independent evaluation of a novel peptide mimetic, brilacidin (PMX30063) for ocular anti-infective. J Ocul Pharmacol Ther 32(1): 23-27.

56. Saravolatz LD, Pawlak J, Johnson J (2012) In vitro activities of LTX109, a synthetic antimicrobial peptide, against methicillin- resistant, vancomycin-intermediate, vancomycin resistant, daptomycin-nonsusceptible and linezolid non-susceptible Staphylococcus aureus. Antimicrob Agents Chemother 56(8): 4478-4482.

57. Gonzales FP, Felgenträger A, Baumler W (2013) Fungicidal photodynamic effect of a twofold positively charged porphyrin against Candida albicans planktonic cells and biofilms. Future Microbiol 8(6): 785-797.
ISSN: 2574-1241

DOI: $10.26717 /$ BJSTR.2018.07.001536

Michael AB Naafs. Biomed J Sci \& Tech Res

(C) (P) This work is licensed under Creative

Submission Link: https://biomedres.us/submit-manuscript.php

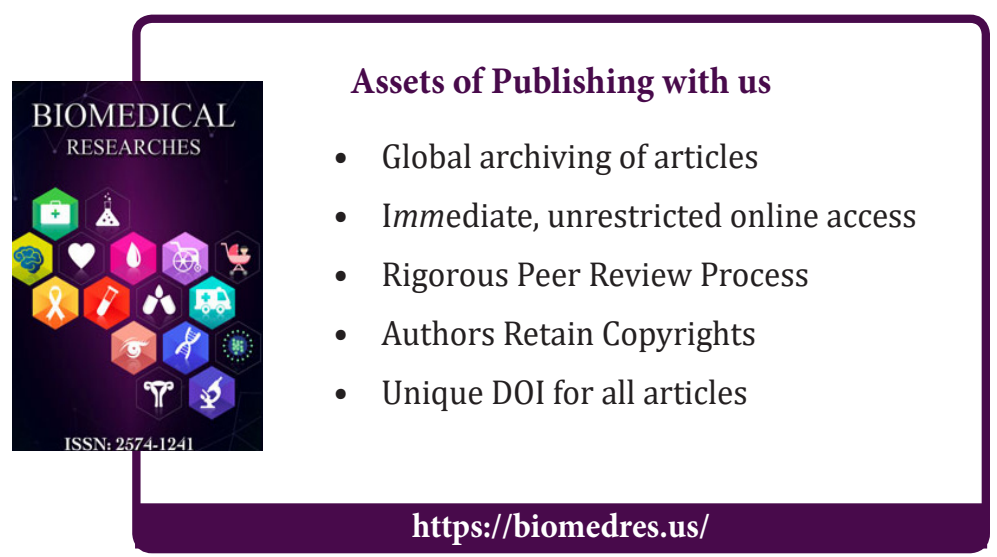

